\title{
Circumferential fusion for spondylolisthesis in the lumbar spine
}

\author{
Robert F. Heary, M.D., AND Christopher M. Bono, M.D. \\ Department of Neurosurgery, The Spine Center of New Jersey, University of Medicine and Dentistry \\ of New Jersey, Newark, New Jersey; and Department of Orthopedic Surgery, Division of Spine \\ Surgery, University of California School of Medicine, San Diego, California
}

\begin{abstract}
There are many options for the surgical treatment of lumbar spondylolisthesis, including anterior and posterior techniques. Among the most versatile is a $360^{\circ}$ fusion. In consideration of the added risk of morbidity of two procedures, circumferential fusion leads to the highest fusion rates. This is particularly useful for patients at high risk for pseudarthrosis, such as patients with diabetes, posttransplant recipients, and those in whom fusion procedures have failed. Likewise, a $360^{\circ}$ fusion may also be useful in achieving fusion in biomechanically disadvantageous situations, such as at the L5-S1 level or with high-grade subluxation. The options for $360^{\circ}$ fusion are many and are determined, among other factors, by surgical pathology and surgeon preference. Standard open techniques are still considered the gold standard, although newer less invasive methods of circumferential fusion are being used more frequently. The operating surgeon must have a thorough knowledge of all available maneuvers for critical and effective decision making.
\end{abstract}

KEY WORDS • spondylolisthesis • fusion • circumferential • interbody

Spondylolisthesis is a common radiographic finding in the lumbar spine. Defined as subluxation of one vertebra on the other, it occurs most frequently at the L4-5 and L5-S1 interspaces. ${ }^{7,11,17,20,23}$ Although subluxation can result from various causes, the majority are either degenerative or isthmic types. Degenerative spondylolisthesis is thought to occur secondary to incompetence of a degenerated disc, which then places greater anteroposterior shear stresses on the eventually arthritic facet joints. With time, the complex fails, and the upper vertebra begins to migrate anteriorly onto the lower vertebra. Degenerative subluxation is most common at L4-5, although it may occur at any level. Isthmic spondylolisthesis is the product of spondylolysis. This is a presumed "stress fracture" within the pars interarticularis. Over time, the pars defect can become elongated, as it represents incompetence of the posterior elements. It is most common at the L-5 pars, allowing subluxation of the L-5 vertebra on the S-1 endplate. Again, this occurs only after the disc begins to fail under the increased shear stresses placed on it.

Abbreviations used in this paper: $\mathrm{ALIF}=$ anterior lumbar interbody fusion; DS = degenerative spondylolisthesis; IS = isthmic spondylolisthesis; LIF = lumbar interbody fusion; PLIF = posterior LIF; PLL = posterior longitudinal ligament; TLIF = transforaminal LIF; VB = vertebral body.
The majority of patients with spondylolisthesis are asymptomatic. It is often detected radiographically as an incidental finding. Unless a high-grade subluxation is recognized (Grade III or higher), asymptomatic patients are simply observed. A percentage of patients, however, develop an array of symptoms related to their subluxated vertebrae. Symptoms are usually related to axial back pain and/or the sequelae of neural compression. ${ }^{17,30}$ Pain is thought to arise from a degenerated disc and facet joint complex. Nociceptive neural innervation of the annulus is thought to be a mechanism for pain transmission. ${ }^{6}$ In addition, the subluxated vertebra can cause a forward shift of the weight-bearing axis. These alterations can lead to fatigue of the lumbar paraspinal muscles in an attempt to maintain sagittal balance.

Neurological symptoms are common with spondylolisthesis. ${ }^{11,17,27,30}$ These can be related to a reduction in dimension of the overall spinal canal at the level of subluxation or compromise of the neural foramina. Thus, patients can present with exercise intolerance from neurogenic claudication and/or radicular complaints. It is not uncommon that patients present with a combination of the two, with radicular complaints worse in one leg than the other. Physical examination may demonstrate objective neurological findings, although this is not typical, because neural dysfunction is usually related to exercise. 
With the exception of very high-grade subluxation, the initial treatment of spondylolisthesis is nonsurgical and includes a combination of pain medications (preferably nonnarcotic) and physical therapy. Traditionally, there is an emphasis on flexion exercises and abdominal strengthening. For patients with claudication or radiculopathy, failure of these measures can be followed by selective nerve root or epidural steroid injections. These often give some symptomatic relief, although it is rarely long lasting. Surgical treatment is considered only after all nonsurgical measures have been exhausted.

Surgical treatment of spondylolisthesis typically consists of a fusion procedure with or without neural decompression. ${ }^{21,23,24,30}$ Obviously, decompressive surgery is reserved for those with symptoms that are correlated with radiographic or advanced imaging evidence of neural impingement. Although laminectomy without fusion had been popular at one time, it is well recognized that this can lead to progressive subluxation and inferior clinical outcomes. ${ }^{17,26}$ Fusion is performed to immobilize the subluxated segments. Arthrodesis can be accompanied by reduction of the vertebral sagittal alignment, although this might risk injury to the exiting nerve root. ${ }^{32}$ Because of this, as well as the excellent clinical outcomes produced by in situ fusion, the benefits of reduction remain controversial. Regardless, numerous surgeons perform reduction maneuvers during fusion procedures to restore sagittal alignment. ${ }^{8,23,32}$ These reduction maneuvers are facilitated by the use of instrumentation that most commonly includes posteriorly placed transpedicular screw constructs.

The application of posterior instrumentation for fusion of spondylolisthesis is another controversial topic. At present the gold standard for the surgical treatment of a singlelevel subluxation is noninstrumented posterolateral lumbar fusion. ${ }^{17}$ Recent data, however, suggest higher fusion rates with the use of instrumentation, especially in longterm follow-up studies compared with noninstrumented procedures. ${ }^{11}$ With fusion is the goal, in addition to clinical improvement, it would seem that the use of instrumentation would be advantageous.

Although posterior procedures are more commonly performed, fusion may be achieved by anterior techniques. Anterior lumbar interbody fusion is the most frequently performed method, with various materials, such as allograft, autograft, or biomechanical devices (such as cages)

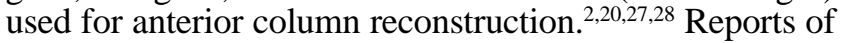
performing ALIF alone for the treatment of lumbar spondylolisthesis are available, with some documenting good results; ${ }^{20}$ however, it is generally recognized that the fusion rates are suboptimal, especially for more than singlelevel arthrodesis; and cage extrusion has been observed with stand-alone anterior constructs. These are especially common when using threaded cages, which probably do not provide sufficient torsional stability to the unstable olisthesed segment, without additional posterior stabilization.

When treating lumbar or lumbosacral spondylolisthesis, the ultimate goal is a solid osseous fusion. For the majority of cases, a posterior fusion, whether instrumented or noninstrumented, is probably adequate; however, these methods are limited by a very definite pseudarthrosis rate, which has ranged between 45 and $91 \% .^{11,17}$ In patients who are at particular risk for nonunion, other methods of fusion should be contemplated. Circumferential fusion has resulted in the highest fusion rates in the lumbar spine, reaching as high as $100 \%$ in some series. ${ }^{2,15,21,36,40}$ Patients at high risk for nonunion include smokers, diabetics, and immunocompromised individuals (those who have undergone organ transplantation). ${ }^{13}$ It is well documented that these patients have a higher rate of nonunion than the general population; thus, it might be prudent to ensure the best possible chance for fusion from the initial procedure. Likely because of greater biomechanical tensile stresses placed across a posterior fusion mass, L5-S1 fusions are difficult to achieve by posterolateral methods alone. Although not always indicated, some patients undergoing a lumbosacral arthrodesis may be candidates for a circumferential procedure. Another high-risk situation is the failed posterior fusion. Again, these patients may be candidates for $360^{\circ}$ fusions. Patients with high-grade subluxation should also be considered for circumferential arthrodesis. Notwithstanding the relative necessity of prophylactic fusion in these cases, posterior fusion alone would cause extremely high tensile stresses. It is also difficult to induce bridging bone in the relatively large distances between the posterior elements of the adjacent subluxated vertebrae.

Circumferential fusion can be performed in a variety of manners. ${ }^{12,13,15,19,25}$ Traditional anterior-posterior fusion includes separate approaches for the interbody and posterior procedures. More recently, all posterior methods of $360^{\circ}$ fusion, such as PLIF or TLIF have gained popularity. ${ }^{4,16,22}$ Some authors consider these to be truly $270^{\circ}$ procedures and fusion rates appear comparable with those in circumferential techniques. ${ }^{36}$ Although extremely useful techniques, they are best performed in patients who have not undergone prior spinal procedures, because dissection through epidural scarring to gain access to the posterior disc space is risky. The anterior component of circumferential fusions can be performed via traditional open (retroperitoneal or transperitoneal), miniopen, or laparoscopic approaches. Likewise, the posterior component can be performed through standard open techniques or with newer percutaneous methods.

The appropriate use of circumferential fusion for the treatment of lumbar spondylolisthesis relies on a clear understanding of its indications, advantages and disadvantages, techniques and applications, and outcomes and complications. It is the purpose of this article to discuss these issues, with a particular focus on the latest available techniques for combined anterior-posterior fusion.

\section{GENERAL INDICATIONS FOR FUSION}

Fusion can be considered in a patient for three main reasons: 1) intractable pain positively correlated with spondylolisthesis in which extensive nonsurgical management has failed, including medications, rehabilitation, and injections; 2) following decompressive laminectomy for lumbar stenosis associated with the subluxated segments; 3) radiographically documented subluxation progression or severe high-grade subluxation greater than $50 \%$ that has a substantial propensity to progress.

\section{Fusion for Pain}

Pain associated with lumbar spondylolisthesis can arise 
from a number of pain generators. When the intervertebral disc degenerates and becomes incompetent, it is a likely contributor. In DS, disc incompetence occurs early in the degenerative cascade, which can manifest as sagittal and/ or rotational deformity. Sagittal instability, or spondylolisthesis, is usually low-grade, static, and slowly progressive. Discogenic pain, without any radiating leg pain or symptoms of claudication, may be a relative indication for fusion if at least 6 months of exhaustive conservative modalities have been attempted. Discography may be used to confirm a single disc level as a significant pain source and indicate a possible favorable result from surgical fusion.

An argument can be proposed supporting circumferential fusion in this situation. Nociceptive innervation of the disc, both anteriorly and posteriorly, has been implicated as a transmitter of pain in degenerative disc disease. ${ }^{6}$ Annulus resection as performed during an ALIF, PLIF, or TLIF could eliminate these nerve endings. Although performing posterolateral fusion methods limit some of the motion at the disc, it is still intact. Micromotion through a solid fusion mass can still theoretically irritate these nerve endings.

Pain in the posterior elements can also arise. Facet joints can become arthritic, especially in DS, whereas IS leaves an intact but ineffective facet joint below. As part of the degenerative cascade, facet incompetence potentiates forward subluxation of one vertebrae onto the next. In IS, pain can be associated with the pars defect itself. When the patient experiences relief with selective injection, this can be a diagnostic clue to this phenomenon. Intractable pain refractory to nonsurgical care associated with either an arthritic facet complex or a lytic defect can be an indication for fusion or, rarely, direct repair.

\section{Fusion After Laminectomy}

Most patients with spondylolisthesis have some degree of back pain; however, this is not always the primary clinical complaint or finding. ${ }^{24,27,34,38,40}$ Because of the subluxation of one vertebra onto the next, patients often present with central stenotic or radiculopathic symptoms. The L-5 root is the most commonly affected in both IS and DS. Patients with central stenosis can present with classic neurogenic claudication, whereas those with foraminal encroachment might present with radiculopathy and severe leg pain. Of course, these can and often do occur concomitantly. If conservative treatment has failed, decompressive surgery may be indicated. Data obtained from a randomized prospective study have indicated significantly better outcome with fusion compared with no fusion after decompressive laminectomy for DS. ${ }^{17}$ Patients' spines that had not fused had a tendency to exhibit additional subluxation and experience less symptomatic relief, whereas those in whom the spine fused had better results. From these and other investigations, it has become clear that fusion should accompany posterior decompression if spondylolisthesis is present, even with Grade I disease.

\section{High-Grade Subluxation}

Most patients present with radiographical evidence of low-grade subluxation (I or II; $<50 \%$ ); however, in some cases patients present with high-grade (III or IV; >50\%) spondylolisthesis..$^{3,7,33,34}$ Despite conservative care, includ- ing abdominal muscle strengthening and physical therapy, these segments are at very high risk for further subluxation. Despite being asymptomatic, patients may be offered early surgical intervention to prevent further subluxation, which may significantly compromise the spinal canal, neural function, or lead to pain. Progression to a Type V subluxation spondyloptosis, should be avoided, because this condition is surgically challenging and can require extensive measures to gain solid fusion. Because of these issues, patients with rapidly progressive or high-grade subluxation should be considered candidates for early surgical fusion.

\section{RELATIVE INDICATIONS FOR CIRCUMFERENTIAL FUSION}

The fusion rate after circumferential fusion is higher than that in stand-alone posterior or anterior procedures, regardless of the method of internal fixation or graft source. Because of this, the relative indications for $360^{\circ}$ arthrodesis to treat spondylolisthesis stem from its extremely high and reliable fusion rate. These include: 1) patient-related factors, such as diabetes, smoking, or immunocompromise; 2) mechanical factors, such as the difficulty in achieving fusion at the lumbosacral junction or multilevel arthrodesis; and 3) postsurgical factors, such as a failed previous fusion, in which the posterior bone healing capability has already proven itself to be incompetent.

\section{Patient-Related Factors}

Diabetes is a known inhibitor of bone healing. Despite controlled sugar levels, it has been demonstrated in animal models that prolonged times are required for long bone callus formation; achieving spinal fusion is challenged by the same factors. Although some authors have documented acceptable fusion rates in diabetic patients who have undergone instrument placement during lumbar procedures, ${ }^{1}$ the general clinical experience has not been so favorable. ${ }^{29}$ Likewise, patients receiving long-term immunosuppressive therapy after solid organ transplantation have compromised bone healing; thus, procedures that would yield the highest possible fusion rate would be most desirable in these patients. The advantages of $360^{\circ}$ fusion must be considered, however, in light of the increased risk of morbidity for an already medically compromised patient. Wound healing and infection risks are important considerations in both diabetic and posttransplantation patients; either issue could influence the decision to add an additional approach and procedure.

\section{Mechanical Factors}

The lumbosacral complex is a transitional area (much like the thoracolumbar and cervicothoracic junctions) and is the focus of greater stresses, particularly sagittal bending forces. The orientation of the L5-S1 disc space is angled caudally, making it more susceptible to shear stresses. In fact, it is thought that this might contribute to the propensity for isthmic subluxation at this level. After posterior methods, greater tensile forces must be resisted by the fusion mass and/or instrumentation. Tensile forces 
are less favorable than compressive loads for fusion and bone healing. In spondylolisthesis, these tensile forces are exaggerated even further than in physiological alignment.

The intervertebral disc spaces, especially in the low lumbar spine, sustain large compressive forces. After discectomy and fusion ALIF, these loads are borne by the reconstruction device, whether it be structural allograft, autograft, or manufactured cage. Along with the large surface area of the exposed bone of the adjacent endplates, these compressive forces are believed to be responsible for the higher fusion rates with interbody compared with posterolateral fusion. As spondylolisthetic spine might be further destabilized after ALIF alone; the addition of posterior fusion and instrumentation yields an even higher rate of fusion. For these reasons, current thinking appears to favor combined anterior-posterior methods for fusion of the lumbosacral junction, becaise it offers the most reliable method of achieving fusion at this high-stress region.

Multilevel fusion is also difficult to achieve, ${ }^{13}$ noninstrumented two-level stand-alone posterior or anterior procedures have resulted in pseudarthrosis rates between 40 and $50 \% .{ }^{20,38}$ Circumferential fusion, is therefore, an excellent option for more than single-level lumbar arthrodesis for spondylolisthesis.

\section{Postsurgical Factors}

Pseudarthrosis is the bane of spinal surgery. It represents the failure of local biological factors to result in bone healing. Failed posterior fusion is common, especially in patients with spondylolisthesis. After noninstrumented procedures, nonunion rates have been reported to be as high as 23 to $40 \% .^{11,17}$ Although clinical outcomes can still be favorable with a stable pseudarthrosis, they can often lead to further instability, pain, and dysfunction. Repair and revision of failed posterior fusion is challenging, because local vascularity has already been compromised by surgical exposure and the quantity of available autograft may be limited due to previous bone harvesting procedures. Circumferential fusion can be a useful method of achieving arthrodesis in such patients because it offers the mechanical and biological advantages of performing a first-time ALIF combined with a revised posterior lumbar fusion. $^{21}$

As discussed, interbody fusion is the preferred salvage method after failed posterolateral arthrodesis. Especially after a laminectomy, postsurgical scarring makes TLIF and PLIF difficult and risky methods of achieving this goal. Staged circumferential fusion is a safer method of achieving interbody fusion in the revision situation, avoiding work through epidural adhesions, while still gaining the benefits of posterior pseudarthrosis exploration and refusion. This should, theoretically, minimize the risk for dural tears during surgery.

\section{ADVANTAGES OF CIRCUMFERENTIAL FUSION OVER STAND-ALONE ANTERIOR OR POSTERIOR METHODS}

The essential defects in DS and IS are in both the anterior and posterior spine. Subluxation cannot occur without failure of both the facets and the intervertebral disc. Over time, the disc and facet joints become incompetent and allow forward subluxation. Pure posterolateral fusion can restore stability only to the posterior elements. A fusion mass between the transverse processes lies behind the axis of rotation of the spine; thus the unstable disc remains a weak link. It can be imagined that even with a solid posterior fusion, the inherent elasticity of the fusion bone can allow stresses to be transmitted to the disc, which potentiates tension forces along the posterior fusion mass.

Likewise, anterior fusion alone can restore only one of the deficient regions of spondylolisthesis. A $360^{\circ}$ fusion has several potential advantages. The endplates of the interbody space offer a more fertile bed for fusion, which likely explains the improved fusion rates compared with pure posterolateral techniques. In addition, the fusion mass is under compression rather than tension. Recent biomechanical data have indicated that the posterior aspect of the intervertebral disc and annulus endure the greatest amount of compressive stresses under load-bearing conditions. ${ }^{9}$ Furthermore, data suggest that interbody distraction can increase the dimensions of the neural foramina. ${ }^{5,42}$ It must be noted, however, that discectomy necessary for the fusion initially destabilizes the spine at that level. This may help explain the occurrence of cage extrusion in some cases after ALIF alone has been performed to treat spondylolisthesis. Thus, ALIF is best combined with posterior stabilization and fusion when treating IS or DS.

Although the necessity of reducing low-grade subluxation remains to be demonstrated, many surgeons believe improvement of sagittal alignment is important. Initial improvement in the sagittal profile has been reported for anterior constructs alone but they tend to collapse with time. ${ }^{38}$ The placement of posterior instrumentation can be an effective means of spondylolisthesis correction, but it places high stresses on the implants, which can lead to hardware failure. As in adult scoliosis, optimal deformity correction is achieved through anterior release followed by placement of posterior instrumentation and fusion. Resection of the annulus (either posterior or anterior) followed by disc space distraction can facilitate realignment. After this "anterior release," pedicle screw instrumentation can be used for correction with less force. Correction must be performed slowly and steadily, with visualization of the nerve roots to help avoid iatrogenic injury. Furthermore, disc space distraction, as with interbody fusion, can increase the dimensions of the neural foramina, resulting in indirect decompression. ${ }^{5,42}$

There are a number of technical advantages to $360^{\circ} \mathrm{fu}-$ sion compared with other methods. Larger single grafts or cages can be placed centered within the disc space compared with posterior methods of interbody fusion. Using TLIF, a single central device can be placed; however, it is obligatorily much smaller than what can be inserted through an anterior approach. The PLIF method usually consists of insertion of two smaller devices side by side. These devices may be biomechanically inferior to larger anteriorly placed cages or grafts. In addition, device insertion does not require neural retraction, which is a particular problem with the PLIF procedure. Although retraction is minimized with TLIF, excessive unilateral bone resection is a potential disadvantage compared with circumferential methods. 


\section{DISADVANTAGES OF $360^{\circ}$ FUSION}

Because of the nature of being two procedures instead of one, circumferential fusion adds to the surgical risk, patient morbidity, and potential complications for the benefit of increased fusion rate. Recent comparisons of circumferential fusion compared with $270^{\circ}$ procedures (TLIF/PLIF) have indicated longer operating times, increased blood loss, higher costs, and longer hospital stays with the former. ${ }^{16,36}$ Interestingly, fusion rates were not significantly different between the procedures, raising doubt about whether the line of distinction between the 270 and $360^{\circ}$ procedures is justified. For the purposes of this paper, PLIF and TLIF will be discussed among other methods of circumferential fusions, although they will be explored in more detail elsewhere in this issue.

\section{Surgical Options for $360^{\circ}$ Fusion}

Traditional methods for circumferential lumbar fusion include open anterior and posterior procedures. These may be performed on the same day during a single period of anesthesia or staged with a planned time interval. Newer methods of approaching the anterior lumbar spine have been developed, including mini-open and laparoscopic techniques. Similarly, endoscopic approaches have been performed in which percutaneously placed pedicle screws have been used for posterior fusion. Although the addition of neurological decompression usually necessitates the use of formal open approaches, techniques of microdecompression in low-grade DS have been advocated. ${ }^{30}$ Advances in posterior fixation of the spine have also taken place, including various designs of pedicle screw, translaminar facet screws, and artificial ligament reconstruction, which may be used as part of a combined $360^{\circ}$ fusion. Circumferential fusion for high-grade subluxation is particularly challenging. Inventive methods, such as spanning pediculovertebral body screws or strut grafts have been successfully used. . $^{3,14}$

\section{Anterior Options}

Anterior Discectomy and Fusion. The anterior lumbar spine can be approached through a formal open retroperitoneal, miniopen, or laparoscopic exposure. It is the authors' preference to perform the miniopen exposure, because it minimizes the size of the incision, has less tendency to conflict with the posterior wound, and enables the use of devices placed via the straight anterior approach. The addition of a $360^{\circ}$ self-retaining retractor attached to a circular frame can be useful for this maneuver. Laparoscopic approaches for lumbar discectomy have been reported, although not specifically in patients with spondylolisthesis. ${ }^{28}$ Whereas the complication rate appears to be higher than with open techniques, the difficulty posed by a spondylolisthetic deformity might only worsen matters. ${ }^{44}$ For this reason, the authors believe that there are very limited indications for laparoscopic discectomy and fusion for DS or IS.

The steps to discectomy are the same regardless of the approach used. First, the anterior VB and their respective discs and anterior longitudinal ligament must be adequately and safely visualized. The level of the bifurcation of the great vessels can vary between L-3 and L-5. The surgeon must decide intraoperatively if work is more safely performed lateral to the iliac vessels or between the bifurcation. Often, if exposure of L4-S1 is necessary, the vessels must be mobilized enough to allow for work both between and lateral to the these structures. Careful and delicate retraction is crucial to avoid iatrogenic injury.

Discectomy does not usually require ligation of the iliolumbar vessels. In some situations, they are sacrificed to allow adequate mobilization of the iliacs for safe visualization. Nevertheless, care must be taken when dissecting proximal and distal to the disc space. The disc is then incised along the endplates and removed with large rongeurs and curettes. The lateral aspects of the annulus can be left intact when the disc is approached directly anteriorly. If dissection is performed lateral to the vessels, the anterior and far-contralateral aspects of the annulus can remain in place. These can help contain the graft and prevent extrusion.

Anterior Decompression. If decompression is indicated, it is usually performed through a posterior laminectomy. This technique allows excellent visualization of the cauda equina and nerve roots. In some situations, an anterior decompression might be desirable. In the authors' experience, substantial osteophytes projecting into the spinal canal from the posterior endplates are difficult to address posteriorly. Using similar techniques to those used in an anterior cervical discectomy, these osteophytes can be resected via the disc space. This is performed by carefully removing all disc material down to the PLL. Usually, the PLL need not be incised or resected as the offending elements are on the ventral surface of this structure. A Kerrison rongeurs or small curved curette can then be carefully used to remove the osteophytes from the posterior lip of the VB. The nerve roots can be indirectly decompressed by cautiously excising the posterolateral aspects of the disc by using a Kerrison rongeurs. Direct visualization of the nerve roots requires extensive removal of the lateral annulus and is usually not performed via this approach. In addition, the absence of the PLL in this region does not offer the same "protection" as it does for the midline neural elements during osteophyte resection. In the authors' opinion, direct root decompression is better treated by performing a posterior laminectomy and foraminotomy.

Anterior Column Reconstruction. The anterior column must now be reconstructed. The disc space can be distracted using a lamina spreader or by extending the table at the break, which should be positioned at the approximate level of the operative disc space. The endplates should be left intact, because this helps maintain compressive strength and prevents graft or cage settling; however, all cartilaginous material must be removed. Several small "punch holes" can be created using a high-speed burr drill or small angled curette to allow access to the underlying bleeding cancellous bone. The disc space dimensions are then measured, including length, width, and height for optimal sizing of the reconstruction cage or graft.

Numerous options exist. A large piece of tricortical iliac crest or fibula can be harvested to be interposed between the endplates; however, this method is limited by donorsite morbidity and the unreliability of the quality of bone, especially in older osteopenic patients. Allograft is a vi- 
able alternative and is probably the most popular method of anterior reconstruction in the lumbar spine used by surgeons in the United States. Precontoured, presized femoral ring allografts are available from many implant companies. These are large pieces of cortical bone that are optimally placed centered within the disc space. The anterior approach is ideal for placement of such a device. The disadvantages of the use of ring allograft are the possibility, albeit extremely low (one/1.5 million for human immunodeficiency virus) of disease transmission, as well as the difficulty monitoring incorporation with the local bone. The elastic modulus mismatch between the cortical graft and the cancellous VB is also a possible culprit of settling, although this is true with the use of any rigid device. Thus, maintenance of the integrity of the vertebral endplates becomes critical.

Titanium mesh cages are being used increasingly throughout the anterior column of the spine. Initially developed by Harms, similar cages are available from most of the major spinal implant companies. The use of titanium, in addition to being magnetic resonance compatible, is closer to the modulus of bone than stainless steel. This being true, it is still exponentially higher than that for cancellous bone. The advantages of titanium mesh are that it is available in numerous diameters and can be cut to the exact length needed. One large cage can be placed, or two smaller cages can be inserted. Because it is packed with bone harvested either locally or from the iliac crest, it offers the biological advantages of autograft with the mechanical stability of metal. The disadvantage of these devices is that they can be easily deformed during insertion if the size is not correct. Ideally, the disc space should be distracted as wide as possible so that the cage slides in with minimal effort and then the distraction is released, allowing the teeth at the ends of the graft to bite into the endplates. These sharp teeth can cause iatrogenic injury to surrounding vessels and structures. They must be visualized during the entire process and kept clear of all vital structures.

Numerous types of metallic threaded cages have been created over the past decade. These cages consist of a hollow dowel-type metallic implant that can thread into the disc like a screw. Preparation of the disc space for these implants consists of a much more limited discectomy performed with power reamers. The annulus and nucleus are removed at the same time with these tools, creating a channeled hole to fit the device exactly. Cage designs vary, with some being implanted from the anterior aspect, whereas others are designed for lateral insertion. A hypothetical advantage of laterally inserted cages is the ability to restore lumbar lordosis. This is achieved by insertion of a slightly smaller cage posterior to a larger cage. Recent investigations have not demonstrated this advantage. Tapered anteroposterior cages have been designed to achieve similar goals, although their efficacy remains to be demonstrated. Again, cages are packed with large amounts of harvested autograft bone.

Carbon fiber cages have been developed by Brantigan and Steffee. ${ }^{4}$ Currently available designs require the insertion of two side-by-side devices, which can be inserted through an anterior (recommended) or lateral approach. Several advantages of these devices are touted. First, the carbon fiber design has a broad surface, maximized by the use of two cages. In addition, the inherent elastic properties of the material are probably closer to those of corticocancellous bone than either femoral rings or titanium cages. These factors might minimize cage settling. A further advantage is related to its radiolucency. Packed with autograft, the device enables easy visualization of the maturation (or lack thereof) a fusion mass within the disc space. Like any biomechanical device, its disadvantages include the fact that it is a foreign body. Although there are currently no reports of abnormal tissue response with the use of these cages in the spine, the use of carbon fiber elsewhere in the body, such as in artificial anterior cruciate ligament reconstructions, has been associated with the development of chronic destructive inflammatory reactions. Long-term follow up and post mortem examinations will be useful in answering these questions.

The authors prefer to use a femoral allograft ring packed with harvested autogenous cancellous bone. Autograft can be obtained from a 1-cm incision made over the anterior crest; then, a small "trap door" of crest is opened using a curved osteotome. This trap door is lifted up, allowing access to the cancellous bone between the two tables, and then shut and tamped back into place. The size of the incision and maintenance of the crest contour appears to limit postoperative pelvic pain.

The femoral ring is usually a reasonable size to fit between the large lumbar or sacral endplates. In addition, the ring can be wedge shaped to enable fine contouring of lordosis, especially at the lumbosacral junction. This can be measured preoperatively to restore alignment and disc space height. The authors' experience using commercially available grafts has been favorable. Although freshfrozen graft is another viable option, achieving the correct graft size and shape is a more time-consuming test of one's carpentry skills. After the graft is in place, distraction is released and the table is flexed back to a neutral position. A nerve hook can be used to ensure adequate space between the graft and the dura. Intraoperative visualization and obtaining a lateral radiograph can help assess the reduction of the subluxation. Often, this is not completely accomplished until posterior instrumentation has been placed. A Kocher or other large clamp may be used to test the security of the graft in its interspace.

Anterior Instrumentation. The placement of anterior instrumentation is controversial in the lower lumbar spine. With a planned posterior surgery and fusion, anterior instrumentation is probably superfluous. If the graft appears to be at particular risk for extrusion, an "antikick" plate or interference screw can be placed. In addition, if further correction is planned via the posterior approach, the plate can help prevent dislodgement during the reduction maneuver. The technique involves a small plate that is screwed into the cranial or caudal aspect of the VB so that the plate overhangs into the operative disc space. This helps prevent the graft from displacing anteriorly. Care is taken to avoid violating the adjacent disc space with the screw. Alternatively, an interference screw with a large washer can be used instead of the plate. Constructs that span the disc space are not usually warranted or recommended. It is critical that the implant be as low profile as possible. Jutting edges from misplacement or migration 
can endanger nearby structures. Because of these risks, the use of anterior instrumentation must be carefully considered in only those cases at very high risk for graft displacement.

Posterior Options. If it is decided that posterior surgery will not be performed on the same day as an anterior procedure, careful transfer of the patient to a flat rotating (RotaRest) or inclinable (Nelson) bed is advised for the interoperative period. This prevents decubitus breakdown as best as possible. Adequate nutrition, whether enteral or parenteral, should be administered during this interim to avoid malnutrition. Wound healing and postoperative infections are best prevented by keeping an absolute lymphocyte count of at least 2000 cells $/ \mathrm{mm}^{3}$ or a serum albumin level of more than $3.5 \mathrm{~g} / \mathrm{dl}^{37}$

If posterior surgery is planned for the same day, the patient is carefully logrolled as a unit onto a second operating table. The authors prefer to use a Jackson (OSI, Union City, CA) spine table with chest, anterior iliac, and proximal thigh bolsters. Avoidance of extreme lordosis helps to minimize the likelihood of graft displacement. Being completely radiolucent, this table allows excellent fluoroscopic visualization of the spine, if this is needed.

Posterior Decompression. Decompressive laminectomy can be performed if indicated. When treating a patient with low-grade spondylolisthesis, this is usually straightforward and not dissimilar to performing laminectomy for degenerative lumbar stenosis not associated with spondylolisthesis. Unreduced high-grade subluxation can be more challenging. The orientation of the nerve roots in relation to the pedicles and the facet joints can be altered. The cauda equina can be deformed by acute bends within the canal, placing it at greater risk for injury during removal of the lamina. When performing laminectomy in a spine with IS, the pars defect should be visualized, because the defect is often covered by hypertrophied fibrous-osseous material, which represents the chronic reaction to mechanical stresses. The Gill-type procedure involves removal of the entire posterior arch. Although it can be removed en bloc, this may risk dural tear from unrecognized adhesions. A piecemeal laminectomy can be more safely performed. All resected bone is cleaned and saved to augment the posterior fusion. After central decompression, the foramina are inspected and decompressed. This is aided by medial facetectomy performed by undercutting the facet joints with an osteotome. Because the decision to perform fusion has already been made, there is less concern about maintaining the integrity of greater than $50 \%$ of the facet joint complex. Finally, nerve root decompression is assessed using a blunt probe.

Methods of "microdecompression" of stenosis associated with DS have been described. ${ }^{30}$ These involve a small (2-in) midline incision with unilateral dissection of the paraspinal muscles. After removal of the inferior aspect of the superior lamina and superior border of the inferior lamina, the ligamentum flavum is removed. Enough caudal lamina is removed to visualize the pedicle. A medial facetectomy can then be performed, and the nerve root can be assessed with a blunt probe. Proponents advocate this method only for decompression in patients with unilateral leg symptoms associated with Grade I subluxation.
Posterior Instrumentation. Although the necessity of placement of posterior instrumentation is still considered controversial for primary posterolateral fusion alone for spondylolisthesis, it has been demonstrated to increase fusion rates. ${ }^{11,12}$ With the indications for a $360^{\circ}$ fusion in patients at high risk for pseudarthrosis, such measures to optimize the likelihood of solid union should be undertaken. Furthermore, anterior discectomy, regardless of how secure the graft or cage fit, can potentially destabilize an already unstable spinal segment. Because solid fusion can taken as many as 2 years to mature, the deformity can progress if not internally fixed. Thus, we routinely use posterior instrumentation to stabilize circumferential fusions.

Open Placement of Pedicle Screws. Posterior transpedicular instrumentation is the most popular stabilization method for DS or IS. It is the authors' practice to perform this using intraoperative fluoroscopic guidance. The pedicle is visualized first on the anteroposterior view. The pedicle center is marked, and a burr or drill is used to create an opening in the cortex. The pedicle finder is then advanced into the VB, guided using both lateral and anteroposterior views. Screw width should be decided based on preoperative imaging, such as computerized tomography or magnetic resonance imaging. Screw length is measured intraoperatively. After placement of all appropriate screws, a rod is contoured in slight lordosis. As the cranial vertebra is subluxated anteriorly on the caudal one, the upper pedicle screws will be displaced anteriorly in relation to the rod. If in situ fusion is preferred, then the rod is contoured to fit easily into the screws. The construct is then compressed and tightened adequately to secure the anterior graft or cage. The advantages of the use of the pedicle screw over other methods of fixation is that it can be used after removal of the posterior neural arch without compromising any strength. Other methods, such as hooks or translaminar/transfacet screws, are not possible at decompressed segments.

Reduction can be performed using a variety of methods. The mobility of the subluxated segment can be assessed by pulling posteriorly on the upper screws. If it appears to be relatively mobile, lordotically contoured rods can first be loosely fitted into the cranial screws. Subsequently, the rods are reduced into the lower screws. Alternatively, the contoured rod can be attached to the screws with the convexity of the curve pointing medially. Before final tightening, the rod can be slowly rotated to place the convexity anteriorly. Rod rotation places less stress across the pedicle screws than in situ bending, which is another option to restore lordotic alignment. ${ }^{43}$ Once acceptable correction is confirmed by direct visualization and intraoperative imaging, the construct is finally tightened according to manufacturers' specifications. It must be noted that the exiting nerve roots should be visualized during any reduction maneuver.

Percutaneous Placement of Pedicle Screws. The demand for less invasive surgical procedures continues to increase. Spine surgery has seen the advent of endoscopically guided scoliosis correction, disc excision, and stenosis decompression. Accordingly, methods for the percutaneous placement of lumbar pedicle screws have been devised. Using fluoroscopic guidance, cannulated screws and guidewires can be inserted into the pedicles. Making 
an incision between the two screw holes, and a separate stab wound caudally, a plate or rod is introduced into the screws. The posterolateral elements are videoscopically decorticated and bone graft is implanted. The use of such techniques is just recently being reported, with no known clinical series of fusions being performed for spondylolisthesis. In the authors' opinion, the efficacy of these methods for IS or DS stabilization would be only in mild Grade I subluxation, in which the anatomy and orientation is near normal. The theoretical advantages of this method are decreased blood loss, shorter hospital times, and smaller incisions. The overall clinical benefit remains to be demonstrated. It does represent a growing trend toward minimally or less invasive methods or surgery. Combined with a laparoscopic or miniopen anterior fusion, a $360^{\circ}$ fusion can be achieved through remarkably small incisions that yield little evidence that an extensive procedure was performed.

Translaminar/Transfacet Screws. Transpedicular screw/rod constructs provide rigid fixation. This is optimal in unstable spines, as well as after performing wide laminectomy of a stenotic spondylolisthetic segment. In low-grade subluxation that do not require extensive decompression, less rigid methods can be considered. Translaminar/transfacet screws can be used to stabilize the lumbar or lumbosacral spine in patients with intact laminae. Long fully threaded cortical screws are introduced into the lamina from a starting portal on the contralateral side of the spinous process. Angling toward the ipsilateral facet joint, the screw travels through the lamina and crosses the facet, with its tip embedded into the transverse process. Surgeons who use this technique have reported a fusion rate as high as $94 \% .{ }^{18}$ Furthermore, they report the ability to perform limited spinal canal decompression by undercutting the lamina and facet joint, although we are not familiar with technique and cannot advocate its use for decompression of spondylolisthetic stenosis. Because fixation relies on achieving purchase across opposing surfaces of a facet joint, fixation of high-grade subluxation would be inherently impossible unless substantial reduction could be achieved. Likewise, facet immobilization below the pars defect in IS would be ineffective. Thus, in our opinion, the use of translaminar/transfacet fixation with $360^{\circ}$ fusion in DS should be reserved for patients with Grade I subluxation that do not require neural decompression. To our knowledge, there are no available reports of this method of fusion for spondylolisthesis.

Artificial Ligament Reconstruction. Novel methods of nonrigid fixation of the lumbar spine have been devised. The Leeds-Keio artificial ligament has been used to stabilize fusions in $\mathrm{DS}^{31}$ The device is passed through a channel created in the posterior VB through the pedicles of the upper vertebra and then the ligament is looped around the spinous process of the inferior segment. The ligament is placed under tension, and a bone plug placed into the pedicles is used for fixation. Standard posterior or posterolateral fusion can then be performed. While the success of the technique relies on the spinous process of the caudal level, a laminectomy cannot be performed there; however, reconstruction would still be possible after laminectomy of the cranial lamina. If sufficient mobility exists, placing tension on the graft can reduce the sublux- ation. Although this method seems a reasonable adjunct to ALIF to achieve $360^{\circ}$ fusion, in an extensive review of the literature, no reports on the use of this method as part of a circumferential fusion could be found. Although technically possible, rigid (pedicle screw) or semirigid (translaminar/facet screws) fixation appears to be more suitable for use with combined anterior-posterior fusion of spondylolisthesis in our opinion.

Open Posterolateral Fusion. Initial dissection should be performed laterally to expose the transverse processes at the levels to be fused. If lumbosacral fusion is planned, then the L5-S1 facet joint is exposed as well as the superior aspect of the sacral ala. The facet joints between all intended fusion levels are cleared of all soft tissue. The capsule is removed and the cartilaginous surfaces are debrided using a small burr. With careful retraction of the paraspinal muscles, the transverse processes, lamina, and spinous processes (if present) are decorticated until a bleeding bone surface is exposed. Large amounts of autogenous bone graft are then placed over these exposed bone surfaces and pushed into place with a wet sponge. Deep retractors are removed, followed by the sponge, leaving the bone graft in its intended place.

The PLIF and TLIF Techniques. Conceptually similar, PLIF and TLIF share common surgical principles. A laminectomy or laminotomy must be performed to expose the neural elements at the level of fusion. Either method can be used with or without a formal neural decompression. This should be performed based on clinical evidence of neurogenic claudication or radiculopathy. Perhaps the safest method is to perform a complete laminectomy at the level of fusion. Performing TLIF necessitates additional resection of the facet on one or both sides. This helps avoid excessive retraction of the dura to gain access the posterior disc space. Of note, this is a more destabilizing maneuver and optimally should be supplemented with posterior instrumentation.

A standard posterior annulotomy is then created using a scalpel, and discectomy is performed. The void is then measured for device sizing. When using prefabricated cages, templates can be inserted to ensure proper size and fit. If autograft iliac crest is to be used, the surgeon has a choice of tricortical or bicortical specimen. Recent evidence suggests better maintenance of disc space height when tricortical graft material has been used. ${ }^{39}$ If a prefabricated cage, metallic or carbon fiber, is used, it should be packed with autogenous cancellous graft harvested from the iliac crest or local bone from the laminectomy. The graft is then tamped into place. Because of space constraints, a single large interbody device is difficult to implant without dangerous retraction of the neural structures; thus, two smaller devices are inserted. These can be inserted through two separate annulotomies on either side of the disc space or through the same side. With the latter method, the first is inserted into the disc space and carefully moved to the contralateral side by using curved tamps. The second implant is inserted adjacent to the first. A potential pitfall while placing two devices is inadvertent migration of the first while the second is being placed. Because of these possibilities, the authors routinely use fluoroscopic guidance to visualize the devices during insertion and confirm acceptable positioning. Optimally, the 
graft or cages should be countersunk approximately 2 to 3 $\mathrm{mm}$ from the posterior VB to avoid iatogrenic compression of the cauda equina.

Postoperative Care. After completion of both the anterior and posterior procedures, the patient should be mobilized as soon as possible. The effects of bracing on fusion rate are presently unclear. With an instrumented $360^{\circ}$ arthrodesis, a brace probably does not add much benefit. Despite this, many surgeons feel more comfortable placing the patient in some type of rigid external immobilization device; unless the residual deformity is severe, an off-the-shelf lumbar orthosis is usually sufficient. A thighextension can be added if lumbosacral fusion was performed. Brace placement can be continued for approximately 3 months, after which the patient is slowly weaned off the device.

Ambulation is encouraged as soon as the patient can tolerate it. Diet is resumed when bowel sounds become present. If a postoperative ileus develops, alternative methods of nutrition should be considered. Muscular reconditioning is an important component of long-term rehabilitation and should begin while the patient is in the hospital.

\section{RESULTS}

Despite its apparent efficacy, there are few dedicated reports on circumferential fusion for spondylolisthesis. Kim, et al., ${ }^{24}$ retrospectively reviewed their surgical results in 89 consecutive cases of adult spondylolisthesis. In patients with isthmic subluxation, posterolateral fusion resulted in a $70 \%$ rate of fusion, whereas a combined technique yielded an $88 \%$ rate; however, these results were not statistically significant because of the low numbers of patients. In patients with DS, the overall fusion rate was $95 \%$. Patients with a solid fusion, regardless of the technique performed, had a statistically better clinical outcome. The authors did not report on the influence of circumferential fusion in degenerative spondylolisthesis cases. The investigators concluded that in patients at high risk of pseudarthrosis, a $360^{\circ}$ fusion should be performed. Unfortunately, the fusion device (autograft, allograft, or cage) was not reported. Although the majority of patients $(78 \%)$ had a Grade I or II subluxation, the grade of subluxation is unclear in the patients who had undergone a $360^{\circ}$ fusion. Postoperative sagittal alignment was not reported.

In a multicenter study, Gertzbein, et al., ${ }^{12}$ reported on fusion rates after staged, combined lumbar anterior-posterior fusion. An allograft strut was placed anteriorly and secured by an antikick screw, and semirigid pedicle screw instrumentation was placed posteriorly. The authors reported an overall $97 \%$ fusion rate. Unfortunately, they did not break down results according to diagnosis, although $33.4 \%$ of their patients had DS. Postoperative alignment was not detailed. Interestingly, patients with spondylolisthesis exhibited substantially better clinical outcomes than those treated for degenerative disc disease. In a subsequent study, Gertzbein, et al., ${ }^{13}$ reported a $100 \%$ fusion rate in 51 patients with degenerative disc disease without spondylolisthesis.

Grob, et al., ${ }^{15}$ reported their findings in 58 patients who underwent circumferential fusion for spondylolisthesis. Most patients (47 of 58) had Grade III or higher subluxation. Although all underwent a staged anterior-posterior fusion, $62 \%$ were performed during the same anesthesia time and $38 \%$ were delayed. The anterior construct consisted of a tricortical iliac crest graft with or without instrumentation. Anterior instrumentation consisted of a long screw and washer directed from the cranial vertebra into the body of S-1. Posteriorly, numerous constructs were used, although pedicle screw and plate was the most common. In contrast to other studies, postoperative alignment was recorded. In patients with Grade I or II spondylolisthesis, less than $10 \%$ reduction was achieved, whereas in those with Grade III and higher, the majority (24 of 38 ) the subluxation was reduced to less than 50\%. Sameday staged procedures resulted in fewer complications and shorter hospital stays. Ninety-seven percent of spines were fused when using these methods. Fusion or lack thereof was not related to the staging of the procedure.

In a retrospective comparison of anterior fusion alone compared with $360^{\circ}$ fusion for degenerative disc disease, Linson, et al., ${ }^{25}$ reported a $100 \%$ fusion rate in the latter whereas in the former the rates were 77 and $83 \%$ fusion for first-time and revision surgery, respectively. Interestingly, all cases of circumferential fusion were performed for previously failed posterior fusions. Posterior instrumentation consisted of pedicle screws.

Rosenberg and Mummaneni ${ }^{35}$ reported their results when using the TLIF procedure in the treatment of Grade I or II spondylolisthesis. The extent of decompression was determined by clinical presentation; however, attempts not to sacrifice the entire facet joint were made. Pedicle screw fixation was used in all cases, and interbody fusion was performed using titanium mesh cages packed with autograft. The only neurological deficit related to the procedure was in a patient with a transient L-5 motor palsy that recovered within 12 months. The authors did not report postoperative sagittal alignment, but did indicate that there was no evidence of cage settling into the endplates seen on follow-up radiographs. Subluxation correction was accomplished by compression across the disc space after cage insertion through the pedicle screw construct. Although the results appear attractive, there are only limited follow-up data in a low number of patients (22), which limits definitive conclusions.

The placement of instrumentation in conjunction with a PLIF has been used to reduce spondylolisthetic subluxation. In an early report on this maneuver, Kamioka and Yamamoto, ${ }^{23}$ used a trapezoidal plate and pedicle screws to lever the cranial subluxation vertebra posteriorly. Interestingly, a total laminectomy was performed at the fused level, which allowed fitting of the plate as well as decompression of the neural elements during reduction. Immediate postoperative alignment and disc height were improved; however, long-term maintenance of these parameters was not achieved. Of note, the plate/screw system was a very early design and was not a fixed-angle device. In addition, the authors did not report the extent of endplate resection, although they did use a "hemicortical" section of iliac crest. Studies have indicated that tricortical graft maintains disc space height better than hemicortical or bicortical grafts. This may also be a factor. 


\section{Complications}

Performing the anterior component of a $360^{\circ}$ fusion carries particular complications. The surgical approach, whether formal open, miniopen, or laparoscopic, endangers important neurovascular structures. The iliac veins and arteries are in intimate contact with the low-lumbar spine. These must be mobilized for adequate visualization and safe work within the disc space. Improper mobilization or retraction can risk vessel tears and hemorrhage and although more easily accessed with formal open procedures, tears during laparoscopic or miniopen approaches can necessitate emergency laparotomy if bleeding cannot be controlled. Postoperative ileus can necessitate for the patient an extended period without an enteral diet. Parenteral supplementation should be considered in these scenarios until enteral feedings can begin.

Retraction and mobilization of the psoas muscle can injure important surrounding neural structures. These include the genitofemoral nerves, the sympathetic plexus, and the exiting nerve roots that form the lumbar plexus. Retrograde ejaculation has been reported in up to $10 \%$ of patients undergoing anterior lumbar approaches. Genitofemoral neurapraxia can result in perineal anesthesia or dysesthesia. Sympathetic plexus injury can result in vasodilation and hyperemia of the ipsilateral lower extremity (paradoxical "ischemia" of the normal contralateral side).

Late complications include pseudarthrosis, although it is exceedingly rare. Allograft fracture has been reported, ${ }^{12}$ as has cage extrusion. In addition to compromising fusion, device migration may create the risk of late injury to surrounding structures. Urgent revision in these situations is indicated, with a vascular or general surgical team present in the event of vascular perforation.

The complications of the posterior component associated with a $360^{\circ}$ fusion are well known. Dural tears can occur during decompression; nerve root injury can occur after reduction of the spondylolisthesis and is best avoided by generous foraminal decompression and visualization. Complications related to the use of pedicle screws during reduction maneuvers include screw breakage, especially if screw placement is suboptimal. In situ rod contouring can weaken the screw-bone interface, contributing to late screw pullout. Cage displacement can also occur after PLIF or TLIF. Uzi, et al., ${ }^{41}$ documented retropulsed cages in two cases requiring emergency revision and stabilization; increased back pain and new onset neurological complaints were the presenting symptoms. In both cases, a PLIF had been performed without posterior instrumentation. On revision surgery, the implants were found to be adequately stabilized after relocation within the disc space by using a compressive pedicle screw construct. The authors recommended careful evaluation of spinal stability after PLIF and the use of pedicle screw stabilization as an adjunct if stability is compromised. The cage was not resized or replaced in either case.

\section{High-Grade Subluxation}

This topic will be discussed separately, because it presents particular challenges to the operating surgeon. Highgrade spondylolisthesis is defined as that with greater than $50 \%$ subluxation of one vertebra on the other (Grade III or
IV). Spondyloptosis is the highest grade, which denotes those in whom $100 \%$ subluxation or greater has occurred. Severe subluxation usually occurs in adolescents or young adults, typically the end-stage of a spondylolytic spondylolisthesis. These are difficult entities to treat, especially by interbody fusion. By definition, the endplates are grossly malaligned, making fit of an interbody graft or device quite challenging. Often times, authors have recommended reduction to provide better endplate contact for improved mechanics of an interbody fusion. Alternatively, in situ fusion, either posterolateral or combined anterior-posterior, has been advocated.

\section{Staged Anterior and Posterior Circumferential Fusion}

Circumferential fusion has been advocated by some authors for high-grade subluxation. Poussa, et al., ${ }^{33}$ compared the results of circumferential in situ fusion with those obtained from reduction and $360^{\circ}$ fusion. Reduction and fusion were performed as a staged procedure with a 2-week delay in an unorthodox sequence. The patients were first underwent posterior fusion by using a transpedicular external fixator. The reduction was achieved slowly, over time, with adjustments of the fixator. Subsequent anterior fusion was performed 2 weeks later. Of importance, no neurological sequelae were observed in the group undergoing reduction. Pain improved comparably in both groups. Correction of sagittal alignment by $11^{\circ}$ was achieved in the reduction group; however, this success was offset by the high rate of complications related to the fixator, such as sepsis and deep infection related to the indwelling pins. Although deformity progressed in the in situ group, comparable clinical results were achieved. Because of the high complication rate, the authors advocated in situ circumferential fusion for severe spondylolisthesis in adolescents.

In an early report of staged anterior-posterior fusion for severe spondylolisthesis in young people, DeWald, et al., ${ }^{7}$ also performed posterior fusion prior to anterior fusion. The authors implanted a Harrington distraction rod from L-1 to the sacrum while posterolaterally fusing only from L-4 to S-2. This construct allowed a slow and gradual reduction of the subluxation. The delayed anterior procedure consisted of interbody fusion between the reduced L-5 vertebra and superior endplate of S-1. The authors routinely removed the posterior rods at 6 to 12 months, at which time exploration revealed a solid fusion in all cases. Correction between 75 and 100\% was reported.

Tiusanen, et al., ${ }^{40}$ compared the results of anterior alone with those of combined approaches in 27 cases. Notably, no instrumentation was used in either technique. Clinical and functional outcomes were comparable in both groups; however, sagittal alignment was significantly better in the group that underwent $360^{\circ}$ fusion. Fusion was successful in all patients, a factor attributed to the patients' young age.

\section{All Posterior $360^{\circ}$ Fusion}

Some have advocated all posterior procedures for circumferential in situ fusion in severe cases. Bohlman and Cook, ${ }^{3}$ as well as others ${ }^{10,34}$ described a technique of transvertebral fusion in which a fibula peg was used after decompression. The procedure entailed insertion of a guide wire from the body of the sacrum upward into the body of 
the cranial VB, under fluoroscopic guidance. An autogenous fibula graft was fashioned to size and inserted through this hole to act as a bone dowel. Posterolateral fusion was supplemented without instrumentation. Fusion rates of 93 to $100 \%$ have been reported. ${ }^{10,34}$ Technical complications of the procedure have included fracture of the fibula peg, although eventual fusion was documented, pseudarthrosis (one of 14 patients in one series), and a dural tear. Temporary peroneal nerve palsy was related to fibula harvest. Overall, back pain was significantly reduced. This technique is suggested as an alternative to staged anterior-posterior fusion for high-grade subluxation, especially in younger patients. ${ }^{34}$

Other surgeons have used pediculo-body screws instead of grafts placed from the posterior approach. The technique involves insertion of a lag screw from the pedicle of the caudal vertebra angled cranially to enter the posteroinferior margin of the upper VB. This effectively created an interbody fusion, taking advantage of the abnormal orientation between the two vertebra. Of importance, this is a method of in situ fusion because the screw cannot be used to reduce the subluxation. Subsequently, a standard posterolateral fusion is performed between the transverse processes. High fusion rates have been reported with good clinical results. ${ }^{14}$ This method does not preclude laminectomy at any level.

\section{CONCLUSIONS}

Numerous techniques exist for the surgical treatment of lumbar spondylolisthesis. The majority of patients can be treated by other methods; however, circumferential fusion can be useful in patients at high risk for pseudarthrosis, revision surgery, and high-grade subluxation. The options for a $360^{\circ}$ fusion are many and are determined, among other factors, by surgical pathology and surgeon preference. Standard open techniques are still considered the gold standard, but newer less invasive methods of circumferential fusion are being used more frequently. A thorough familiarity with all available maneuvers is crucial to critical and effective decision making when treating patients with this difficult clinical entity.

\section{References}

1. Bendo JA, Spivak J, Moskovich R, et al: Instrumented posterior arthrodesis of the lumbar spine in patients with diabetes mellitus. Am J Orthop 29:617-620, 2000

2. Bendo JA, Spivak JM, Neuwirth MG, et al: Use of anterior interbody fresh-frozen femoral head allograft in circumferential lumbar fusions. J Spinal Disord 13:144-149, 2000

3. Bohlman HH, Cook SS: One-stage decompression and posterolateral and interbody fusion for lumbosacral spondyloptosis through a posterior approach. Report of two cases. J Bone Joint Surg Am 64:415-418, 1982

4. Brantigan JW, Steffee AD: A carbon fiber implant to aid interbody lumbar fusion. Two-year clinical results in the first $26 \mathrm{pa}-$ tients. Spine 15:2106-2107, 1993

5. Chen D, Fay LA, Lok J, et al: Increasing neuroforaminal volume by anterior interbody distraction in degenerative lumbar spine. Spine 20:74-79, 1995

6. Coppes MH, Marani E, Thomeer RT, et al: Innervation of "painful" lumbar discs. Spine 22:2342-2350, 1997
7. DeWald RL, Faut MM, Taddonio RF, et al: Severe lumbosacral spondylolisthesis in adolescents and children. Reduction and staged circumferential fusion. J Bone Joint Surg Am 63: 619-626, 1981

8. Diedrich O, Perlick L, Schmitt O, et al: Radiographic spinal profile changes induced by cage design after posterior lumbar interbody fusion preliminary report of a study with wedged implants. Spine 26: E274-E280, 2001

9. Edwards WT, Ordway NR, Zheng Y, et al: Peak stresses observed in the posterior lateral anulus. Spine 26:1753-1759, 2001

10. Esses SI, Natout N, Kip P: Posterior interbody arthrodesis with a fibular strut graft in spondylolisthesis. J Bone Joint Surg Am 77:172-176, 1995

11. Fischgrund JS, Mackay M, Herkowitz HN, et al: 1997 Volvo award winner in clinical studies. Degenerative lumbar spondylolisthesis with spinal stenosis: a prospective, randomized study comparing decompressive laminectomy and arthrodesis with and without spinal instrumentation. Spine 22:2807-2812, 1997

12. Gertzbein SD, Betz R, Clements D, et al: Semirigid instrumentation in the management of lumbar spinal conditions combined with circumferential fusion. A multicenter study. Spine 21: 1918-1926, 1996

13. Gertzbein SD, Hollopeter M, Hall SD: Analysis of circumferential lumbar fusion outcome in the treatment of degenerative disc disease of the lumbar spine. J Spinal Disord 11:472-478, 1998

14. Grob D, Humke T, Dvorak J: Direct pediculo-body fixation in cases of spondylolisthesis with advanced intervertebral disc degeneration. Eur Spine J 5:281-285, 1996

15. Grob D, Scheier HJ, Dvorak J, et al: Circumferential fusion of the lumbar and lumbosacral spine. Arch Orthop Trauma Surg 111:20-25, 1991

16. Hee HT, Castro FP Jr, Majd ME, et al: Anterior/posterior lumbar fusion versus transforaminal lumbar interbody fusion: analysis of complications and predictive factors. J Spinal Disord 14:533-540, 2001

17. Herkowitz HN, Kurz LT: Degenerative lumbar spondylolisthesis with spinal stenosis. A prospective study comparing decompression with decompression and intertransverse process arthrodesis. J Bone Joint Surg Am 73:802-808, 1991

18. Humke T, Grob D, Dvorak J, et al: Translaminar screw fixation of the lumbar and lumbosacral spine. A 5-year follow-up. Spine 23:1180-1184, 1998

19. Humphreys SC, Hodges SD, Patwardhan AG, et al: Comparison of posterior and transforaminal approaches to lumbar interbody fusion. Spine 26:567-571, 2001

20. Inoue $S$, Watanabe $T$, Goto $S$, et al: Degenerative spondylolisthesis. Pathophysiology and results of anterior interbody fusion. Clin Orthop 227:90-98, 1988

21. Jones AA, McAfee PC, Robinson RA, et al: Failed arthrodesis of the spine for severe spondylolisthesis. Salvage by interbody arthrodesis. J Bone Joint Surg Am 70:25-30, 1988

22. Jun BY: Posterior lumbar interbody fusion with restoration of lamina and facet fusion. Spine 25:917-922, 2000

23. Kamioka Y, Yamamoto $\mathrm{H}$ : Lumbar trapezoid plate for lumbar spondylolisthesis. A clinical study on preoperative and postoperative instability. Spine 15:1198-1203, 1990

24. Kim SS, Denis F, Lonstein JE, et al: Factors affecting fusion rate in adult spondylolisthesis. Spine 15:979-984, 1990

25. Linson MA, Williams H: Anterior and combined anteroposterior fusion for lumbar disc pain. A preliminary study. Spine 16: $143-145,1991$

26. Lombardi JS, Wiltse LL, Reynolds J, et al: Treatment of degenerative spondylolisthesis. Spine 10:821-827, 1985

27. Mardjetko SM, Connolly PJ, Shott S: Degenerative lumbar spondylolisthesis. A meta-analysis of literature 1970-1993. Spine 19 (Suppl 20):2256S-2265S, 1994

28. Mathews HH, Evans MT, Molligan HJ, et al: Laparascopic 


\section{R. F. Heary and C. M. Bono}

discectomy with anterior lumbar interbody fusion. A preliminary review. Spine 20:1797-1802, 1995

29. McAfee PC, Cunningham BW, Lee GA, et al: Revision strategies for salvaging or improving failed cylindrical cages. Spine 24:2147-2153, 1999

30. McCulloch JA: Microdecompression and uninstrumented single-level fusion for spinal canal stenosis with degenerative spondylolisthesis. Spine 23:2243-2252, 1998

31. Mochida J, Toh E, Suzuki K, et al: An innovative method using the Leeds-Keio artificial ligament in the unstable spine. Orthopedics 20:17-23, 1997

32. Petraco DM, Spivak JM, Cappadona JG, et al: An anatomic evaluation of L5 nerve stretch in spondylolisthesis reduction. Spine 21:1133-1139, 1996

33. Poussa M, Schlenzka D, Seitsalo S, et al: Surgical treatment of severe isthmic spondylolisthesis in adolescents. Reduction or fusion in situ. Spine 18:894-901, 1993

34. Roca J, Ubierna MT, Caceres E, et al: One-stage decompression and posterolateral and interbody fusion for severe spondylolisthesis. An analysis of 14 patients. Spine 24:709-714, 1999

35. Rosenberg WS, Mummaneni PV: Transforaminal lumbar interbody fusion: technique, complications, and early results. Neurosurgery 48:569-575, 2001

36. Schofferman J, Slosar P, Reynolds J, et al: A prospective randomized comparison of 270 degrees fusions to 360 degrees fusion (circumferential fusions). Spine 26: E207-E212, 2001

37. Stambough JL, Beringer D: Postoperative wound infections complicating adult spine surgery. J Spinal Disord 5:277-285, 1992
38. Takahashi K, Kitahara H, Yamagata M, et al: Long-term results of anterior interbody fusion for treatment of degenerative spondylolisthesis. Spine 15:1211-1215, 1990

39. Takeda M: Experience in posterior lumbar interbody fusion: unicortical versus bicortical autologous grafts. Clin Orthop 193:120-126, 1985

40. Tiusanen H, Schlenzka D, Seitsalo S, et al: Results of a trial of anterior or circumferential lumbar fusion in the treatment of severe isthmic spondylolisthesis in young patients. J Pediatr Orthop B 5:190-194, 1996

41. Uzi EA, Dabby D, Tolessa E, et al: Early retropulsion of titanium-threaded cages after posterior lumbar interbody fusion: a report of two cases. Spine 26:1073-1075, 2001

42. Vamvanij V, Ferrara LA, Hai Y, et al: Quantitative changes in spinal canal dimensions using interbody distraction for spondylolisthesis. Spine 26: E13-E18, 2001

43. Voor MJ, Roberts CS, Rose SM, et al: Biomechanics of in situ rod contouring of short-segment pedicle screw instrumentation in the thoracolumbar spine. J Spinal Disord 10:106-116, 1997

44. Zdeblick TA, David SM: A prospective comparison of surgical approach for anterior L4-15 fusion: laparoscopic versus mini anterior lumbar interbody fusion. Spine 25:2682-2687, 2000

Manuscript received May 20, 2002.

Accepted in final form June 18, 2002.

Address reprint requests to: Robert F. Heary, M.D., UMDNJNew Jersey Medical School, 90 Bergen Street, DOC Suite 8100, Newark, New Jersey 07103. email: heary@umdnj.edu. 